\title{
ESTUDOS DE DINÂMICA MOLECULAR COM COMPOSTOS SELECIONADOS POR TRIAGEM VIRTUAL FRENTE AO COMPLEXO ENZIMÁTICO ANTÍGENO 85 DO Mycobacterium tuberculosis
}

\author{
Elivelton de Jesus Cerqueira'; ${ }^{1}$ Manoelito Coelho dos Santos Júnior²; Isis Bugia \\ Santana $^{3}$ \\ 1. Bolsista PIBIC-Af/CNPq, Graduando em Farmácia, Universidade Estadual de Feira de Santana, e-mail: \\ tomcerqueira2@gmail.com \\ 2. Orientador, Departamento de Saúde, Universidade Estadual de Feira de Santana, e-mail: manoelito@uefs.br \\ 3. Colaborada, Programa de Pós-graduação em Biotecnologia, Departamento de Ciências Biológicas, Universidade Estadual \\ de Feira de Santana, e-mail: isisbugia@uefs.br
}

PALAVRAS-CHAVE: Triagem virtual hierárquica; Mycobacterium tuberculosis; antígeno 85.

\section{INTRODUÇÃO}

A tuberculose (TB) é uma afecção causada pela Mycobacterium tuberculosis (Mtb) que, dentre as doenças infecciosas, é a principal causa de morte no mundo, estimando-se que em 2015 ela tenha acarretado o óbito de 1,4 milhões de pessoas (WHO, 2016). A resistência bacteriana aos fármacos utilizados no tratamento configura um dos principais desafios para o controle da doença (WHO, 2016). Logo, é urgente a busca por novas alternativas terapêuticas no combate a TB.

No sentido de intervir em vias ligadas à virulência da $M t b$, as proteínas antígeno 85 , representadas pelos antígenos 85A, 85B e 85C apresentam-se como alvos promissores para fármacos por catalisar a biossíntese de micolatos, elementos essenciais para a composição da parede celular da bactéria, e por transferi-los ao arabinogalactano (Ronning et al. 2000). Sendo assim, a modulação exclusiva do Ag85C é justificada no fato das proteínas apresentarem sítio ativo altamente conservado, com $100 \%$ de identidade para os resíduos da tríade catalítica (Ronning et al. 2004).

No intuito de buscar potenciais inibidores para o $\mathrm{Ag} 85 \mathrm{C}$, a triagem virtual é empregada para selecionar compostos promissores frente a determinado alvo terapêutico com o intuito de priorizar moléculas com elevado potencial de atividade biológica para os posteriores testes biológicos (Rodrigues et al. 2012). Estratégias como a triagem virtual hierárquica, que associa a triagem baseada no alvo com a triagem baseada no ligante, contribuem para a seleção dessas moléculas promissoras.

Logo, esse trabalho teve como objetivo principal identificar potenciais inibidores frente ao Ag85C, por triagem virtual, e como específicos: selecionar compostos oriundos do banco de produtos naturais; realizar estudos de acoplamento molecular com os compostos selecionados; classificar os melhores compostos ranqueados de acordo a regra de Lipinski; realizar estudos de dinâmica molecular e construir mapas de interação intermolecular entre compostos selecionados e a enzima alvo.

\section{METODOLOGIA}

Inicialmente foi organizado um banco de inibidores com atividade conhecida frente ao Ag85C, no qual, uma parte foi oriunda do banco de dados ChEMBL (Bento et al. 2014), com IC50 $<50 \mu \mathrm{M}$ e a outra do trabalho de Ibrahim e colaboradores (2012), com Ki $<50 \mu \mathrm{M}$. Um banco de compostos naturais (BIOGENICS) foi obtido do ZINC15 (Sterling \& Irwin, 2015). 
A triagem virtual por similaridade química foi realizada utilizando como moléculas de referência os inibidores do Ag85C. A partir de então utilizou-se o programa DataWarrior 4.2.2 (Sander et al. 2015).

Para a realização do acoplamento molecular foi selecionada a estrutura cristalográfica do Ag85C (PDB ID 1VA5), co-cristalizada com o ligante octil tioglicosideo (OGS), o preparo da mesma foi realizado no UCSF Chimera 1.9.

O acoplamento molecular foi realizado com o pacote de programas DOCK 6.7 (Ewing et al. 2001). Com o programa SPHGEN foi construído um conjunto de esferas com base na superfície molecular. A avaliação dos parâmetros do acoplamento molecular foi realizada através da análise do valor de RMSD (do inglês Root Mean Square Deviation). Para pontuação das moléculas foi utilizada a função padrão Grid Score.

Os compostos melhores ranqueados pelo acoplamento molecular foram classificados segundo a regra do 5 de Lipinski no servidor Molinspiration 2012.05 (Ertl, 2010).

O composto selecionado como melhor potencial inibidor foi submetido a simulações por dinâmica molecular (DM) junto ao Ag85C. A minimização inicial dos complexos foi realizada no pacote de programas GROMACS 5.1.2 (Pronk et al. 2013) empregando-se o método de mecânica molecular com o campo de força GROMOS96 53a6 (Oostenbrink et al. 2004). A minimização de energia foi realizada empregando-se os algoritmos Steepest Descent e posteriormente Gradiente conjugado, ambos com e sem restrição.

Após as etapas de minimização de energia, utilizando o integrador Verlet (Verlet, 1967), o sistema foi equilibrado e aquecido à temperatura constante de $300 \mathrm{~K}$ numa simulação de dinâmica molecular (1ns) utilizando o algoritmo LINCS. Em seguida, nas mesmas condições, foi realizada uma simulação de dinâmica molecular de 30ns, sem restrições.

As interações intermoleculares do complexo foram analisadas por meio do servidor Protein-Ligand Interaction Profiler (PLIP) (Salentin et al. 2015) para a construção dos mapas de interações em 3D, tanto para o acoplamento quanto para a dinâmica molecular.

\section{RESULTADOS E DISCUSSÃO}

Na pesquisa realizada no ChEMBL (Bento et al. 2014) selecionou-se cinco compostos, os quais são identificados como: CHEMBL325304, CHEMBL118252, CHEMBL326268, CHEMBL227546 e CHEMBL418868; já no trabalho de Ibrahim e colaboradores (2012) selecionou-se os compostos 13a, 13b e 14.

Os resultados da busca por similaridade demonstraram que para os inibidores retirados do ChEMBL não foram encontrados compostos com similaridade maior que 0,7 ; no entanto foram obtidos 1.363 compostos similares aos inibidores 13a, 13b e 14 .

$\mathrm{O}$ resultado do reacoplamento molecular indicou $\mathrm{RMSD}=1,23 \AA$ para a função de pontuação Grid Score, mostrando sucesso em reposicionar o ligante OGS. Após a realização do acoplamento molecular e aplicação do filtro de Lipinski o composto ZINC000107283125, com energia de afinidade igual a $-84,655426 \mathrm{Kcal} / \mathrm{mol}$, foi designado para os estudos de dinâmica molecular.

Durante a simulação de DM a energia do total do sistema (forma APO e HOLO) manteve-se em torno de $-6,7 \mathrm{~kJ} / \mathrm{mol}$, certificando o enovelamento da proteína durante todo o tempo de simulação. Para a análise da estabilidade estrutural foi possível observar que a forma APO estabilizou a partir de $15 \mathrm{~ns}$ de simulação, apresentando desvio padrão médio para as posições atômicas de 5,6 e uma variação em torno de $2,75 \AA$, enquanto a forma HOLO estabilizou a partir de 11 ns com desvio médio padrão de 2,3 e variação em torno de 2,25 A. Essa maior estabilidade do complexo, em comparação à forma APO, pode ser devido à realização de interações intermoleculares que influenciam diretamente na oscilação das posições atômicas. 
Na verificação da contribuição de determinadas regiões da proteína através do RMSF (do inglês Root Mean Square Fluctuation) da cadeia principal, observou-se que as regiões de alça demonstraram maior instabilidade conformacional em relação às demais estruturas secundárias (Figura 1). A proteína APO apresentou desvio padrão médio para a flutuação dos resíduos de 9,5, já a HOLO de 5,3. Logo, pode-se afirmar que ZINC000107283125 foi capaz de induzir modificações conformacionais, aumentando a estabilidade na área de catálise e diminuindo em regiões próximas, mas que, de modo geral, tenderam a estabilizar a estrutura. Destaca-se ainda que a tríade catalítica (Ser125, Glu229 e His261), na forma HOLO apresentou menores flutuações do que na APO.

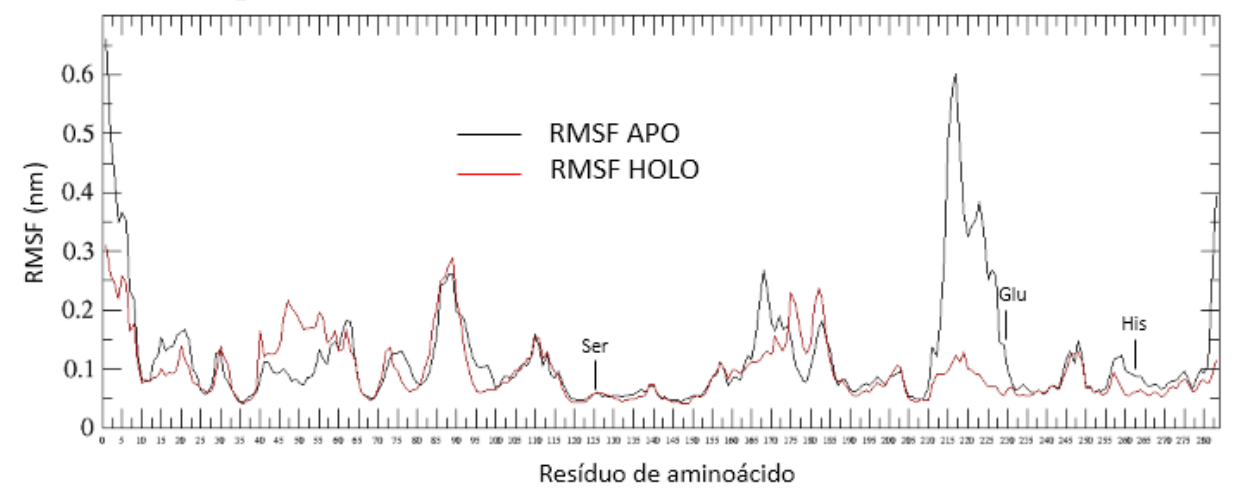

Figura 1 - Representação gráfica do RMSF para a cadeia principal (backbone) da forma APO e da forma HOLO durante a simulação por DM, destacando os resíduos da tríade catalítica.

Com a estrutura representativa da DM foi possível verificar que ZINC000107283125 (Figura 2) realizou interações hidrofóbicas com os resíduos Trp159, Leu162, Leu165, Leu228. Foram verificadas também a realização de ligações de hidrogênio do tipo aceptora com Ser125, Ala225, e Asn222; e do tipo doadora a Asn222.

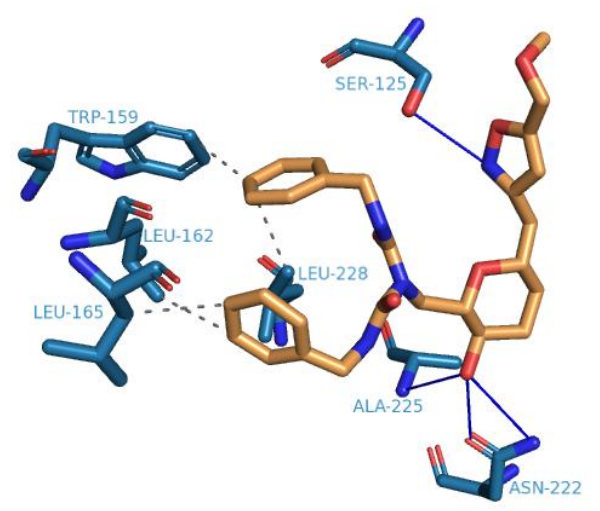

Figura 2: Mapa de interações intermoleculares do composto ZINC85627062 com o Ag85C da DM. Linha preenchida azul: ligação de hidrogênio. Linha tracejada cinza: interação hidrofóbica.

\section{CONCLUSÕES}

A abordagem baseada na triagem virtual hierárquica híbrida pôde, de forma significativa, diminuir o tempo de busca por um potencial inibidor do Ag85C e o custo computacional associado a mesma. A aplicação da similaridade química reduziu o BIOGENICS para aproximadamente $1,5 \%$, e direcionou para seleção de estruturas químicas pertencentes ao um espaço químico biologicamente.

Utilizando a função de pontuação Grid Score, selecionou-se a molécula ZINC000107283125. Com os resultados da DM foi possível verificar o complexo Ag85C/ZINC000107283125 manteve-se estável durante a fase produtiva. Assim, os resultados com os estudos realizados contribuíram para a pesquisa de novos fármacos mais seletivos e potentes frente ao $\mathrm{Ag} 85 \mathrm{C}$ e, possivelmente, às demais proteínas antígeno $85 \mathrm{da} M t b$. 


\section{REFERÊNCIAS}

WORLD HEALTH ORGANIZATION (WHO). 2016. Global tuberculosis report 2016. 20. ed. Geneva: WHO.

RONNING, D. R. et al. 2000. Crystal structure of the secreted form of antigen 85C reveals potential targets for mycobacterial drugs and vaccines. Nature Structural Biology 7(2).

RONNING, D. R. et al. 2004. Mycobacterium tuberculosis Antigen 85A and 85C Structures Confirm Binding Orientation and Conserved Substrate Specificity. The Journal of Biological Chemistry 279(35), 36771-7.

RODRIGUES, R.P. et al. 2012. Estratégias de triagem virtual no planejamento de fármacos. Revista Virtual de Química 4(6): 739-776.

BENTO, A.P. et al. 2014. The ChEMBL bioactivity database: an update. Nucleic Acids Research 42: 1083-1090.

IBRAHIM, D. A. et al. 2012. Design, synthesis, and x-ray analysis of a glycoconjugate bound to Mycobacterium tuberculosis Antigen 85C. Bioconjugate Chemistry 23: 2403-2416.

IRWIN, J.J. et al. 2012. ZINC - A free tool to discover chemistry for biology. Journal of chemical information and modeling (1): 1-40.

SANDER, T. et al. 2015. DataWarrior: an open-source program for chemistry aware data visualization and analysis. Journal of chemical information and modeling 55(2): 460-73.

EWING, T.J.A. et al. 2001. DOCK 4.0: search strategies for automated molecular docking of flexible molecule databases. J. Comput. Aided Mol. Des. 15(5): 411-428.

ERTL, P. 2010. Molecular Structure Input on the Web. Journal of Cheminformatics 2(1).

OOSTENBRINK, C. et al. 2004. A biomolecular force field based on the free enthalpy of hydration and solvation: the GROMOS force-field parameter sets 53A5 and 53A6. Journal of Computational Chemistry 25(13): 1656-76.

VERLET, L. 1967. Computer "Experiments" on Classical Fluids. I. Thermodynamical Properties of Lennard-Jones Molecules. Physical Review, 159(1): 98-103.

SALENTIN, S. et al. 2015. PLIP: fully automated protein-ligand interaction profiler. Nucleic Acids Research 43: W443-W447. 